\title{
A perspective on vaccine evaluation research in Canada: Past and future
}

\author{
DAVID W SChEIFELE, MD, AUBrey J TINGLE, MD, PHD
}

$\mathrm{T}$ HE 1990s WILL BE NOTEWORTHY FOR A RENAISSANCE IN vaccine use, among other major developments in infectious diseases. The technology tree will yield its long-awaited fruit in increasing numbers and variety, providing a cornucopia of new vaccination options for infants and children. Governments intent upon controlling the costs of health care can be expected to seize upon many of these new vaccines to implement new programs of disease prevention. There is little doubt that good vaccines well used are the most cost effective measure in modern medicine. We believe that vaccines represent the very best of high technology because their bottled magic is available to all, even the youngest tyke in the most remote village.

In 1992, change already is afoot. Conjugate vaccines for prevention of Haemophilus influenzae type b infections have made their debut in programs in virtually every province and territory. The innovation of conjugating bacterial polysaccharide to selected protein carriers allowed the age barrier in responsiveness to polysaccharide to be broken. The new programs, beginning at two months of age, can virtually eradicate $H$ influenzae type b infections within a few years. New programs are being organized to deliver recombinant hepatitis $\mathrm{B}$ vaccines to children, protecting them in advance of the peak risk in early adulthood. British
Columbia initiated such a program for all grade 6 students in autumn 1992, the first province to do so.

Many additional vaccines will be licensed during this decade. The list will include a varicella vaccine, inactivated hepatitis A vaccine, oral typhoid vaccine and acellular pertussis vaccines - all products that have already been licensed in some developed countries. Based upon studies now in progress, it is likely that the list will also include rotavirus and respiratory syncytial virus vaccines. The list of candidate products early in development, including human immunodeficiency virus vaccines, is too long to enumerate.

Consumers, especially parents of young children, will expect these new vaccines to be safe and convenient. Willingness to accept the frequent occurrence of minor side effects (as with whole cell pertussis vaccine) or rare risks of serious adverse effects (as with oral poliovirus vaccine) already is diminishing. As less familiar and feared microbes are targeted, parents will demand convenient mixing of new vaccines with established ones - a complex technological challenge. The ability to manufacture all components of a combination vaccine will become paramount, forcing manufacturers into cooperative alliances and eliminating countryspecific products or formulations.

With so many changes predictably ahead, it is timely

Based on an invited lecture to the Canadian Infectious Diseases Society presented by Dr Scheifele on February 3, 1991, at Val David, Quebec

Division of Infectious and Immunologic Diseases, Department of Pediatrics, British Columbia's Children's Hospital and the University of British Columbia, Vancouver, British Columbia

Correspondence and reprints: Dr David W Scheifele, Director, Vaccine Evaluation Center, Professor of Pediatrics, 950 West 28 th Avenue, Vancouver, British Columbia V5Z 4H4. Telephone (604) 875-2422, Fax (604) 875-2635

Received for publication August 14, 1992. Accepted October 17, 1992 
to review the process involved in licensing new vaccines. Is Canada ready for this challenge? Who, specifically, will help to meet it?

\section{PRELICENSURE TESTING OF A VACCINE FOR CHILDREN}

The process of testing a candidate vaccine for a common childhood infection begins in the laboratory with critical appraisal of manufacturing 'chemistry', including parameters such as purity, potency, stability and lot-to-lot consistency. Animal studies follow to assess toxicity of large doses, the detailed nature of the immune response and, where models of infection exist, the ability of vaccination to protect against infection. The manufacturer plays the major role in preclinical studies, but those in animals are often undertaken with academic partners, particularly ones with special expertise in defining immune responses to the pathogen of interest.

Clinical testing of new vaccines follows a prescribed. stepwise sequence that is calculated to minimize risks and closely observed by regulatory agencies of countries where the testing is performed. 'Phase 1' studies involve the first tentative uses in humans, characterized by involvement of small numbers of adult volunteers and emphasis on evaluation of safety. The secondary objectives are dose finding and assessment of immune responses to what is often a secondary stimulus.

With such reassurances, testing enters 'phase 2 ' where studies move stepwise from secondary stimulation of immune responses in older children (assuming the target is a common infection) to studies in the intended age group. Once again, protocols are designed to include just enough subjects to answer the desired question. Over a series of studies such questions include determination of optimal dose and schedule, influence of age and maternally derived antibody on responses, lot-to-lot consistency of immunogenicity and. through all, the continuing close assessment of adverse effects. As confidence in the product increases, more studies are initiated in parallel to assess vaccine performance in genetically diverse populations, in groups at increased risk for the target infection and in conjunction with other vaccines routinely used in the age group to confirm compatibility. These designs are sufficiently alike to permit accumulation of substantial numbers of observations on immune responses and safety. The earliest studied children of the target age are followed over time to assess the stability of immune responses and the need for any booster doses.

'Phase 3 ' is the acid test, the attempted demonstration of protective efficacy. Whereas scores of studies might be done in phase 2 , a single large study is the rule in phase 3 . This is undertaken in an area where disease risk is high, permitting relatively limited enrollment and duration of follow-up. The study environment must be controlled sufficiently so that results will be accurate and generalizable to most countries. The outcome measure can be absolute (vaccine versus placebo) or relative efficacy (where an older vaccine exists for comparison). Design and conduct of the trial must be rigorous to withstand extensive subsequent scrutiny by regulatory agencies and expert advisory groups.

A key part of a phase 3 study is the search for a serological correlate of protection because this becomes the benchmark for assessment of vaccine performance in other populations. Because most phase 3 studies involve thousands of subjects, they also provide a substantial body of safety data.

As the phase 3 study nears completion, the manufacturer generally will undertake at least one safety and immunogenicity trial in each country where licensure will be sought; this famiiiarizes the regulatory agency with the product and can accommodate any unique requirements for licensure (eg, testing in expanded ethnic groups or in conjunction with parochial routine vaccines). While most foreign manufacturers have followed this tradition in Canada, the Bureau of Biologics has no statutory authority to require studies to be done here.

The period following licensure is referred to as 'phase 4'. Post marketing concerns centre on the possibility of rare but serious adverse effects not revealed by the limited scale of prelicensure testing and on the vaccine's ability to prevent infection under 'real world' conditions of use. Post marketing safety studies increasingly are approached like efficacy trials, where one large study is conducted in the country of origin or first major marketplace country, often while licensure is awaited in other countries. Since the outcome measures in these studies are adverse events resulting in doctor visits or hospital admissions, group health organizations with computerized record systems are wellsuited to perform them. Measurement of disease control requires data on large defined populations. Case-control designs are used to estimate vaccine efficacy in routine programs.

Some post licensure issues can take decades to elucidate, such as the duration of protection, need for booster doses, the nature and severity of breakthrough infections, and the level of immunity in a population needed to stop disease transmission.

A new and pressing aspect of phase 4 is comparison of competing products. A corollary of the new biotechnology is that new products will come to market in competing plenty; this will foster price competition and 'survival of the fittest' products, but multiply the already huge costs of product development. Mechanisms must evolve under government sponsorship to compare products 'head to head' as rigorously and uniformly as possible to enable health administrators to identify the best products.

Another pressing issue for newly marketed vaccines 
for children is their integration, when possible, into combinations with routinely administered vaccines. Avoiding multiple injections per visit or extra visits is a high priority but constructing combination vaccines without loss of potency of any component technically is challenging. From a consumer's viewpoint, there is room for concern that combination 'packages' will not always include the best among new products because some manufacturers will be better positioned than others to offer combinations.

\section{PARTNERS IN THE EVALUATION PROCESS}

Clinical evaluation of new vaccines requires cooperation between the regulatory agency, vaccine manufacturer and clinical investigators, and each has a unique role. The regulatory agency is the referee, protecting the interests of participating subjects and future vaccinees above all else. In Canada, the regulatory function is served by the Bureau of Biologics within the Health Protection Branch of Health and Welfare Canada. Despite limited staffing, the Bureau of Biologics has earned a reputation for high standards. The United States' Food and Drug Administration accepts studies performed in Canada as equivalent to American ones, the only foreign studies given this status.

Among the five vaccine companies active in Canada, two produce some or all of their products here while three are vendors with manufacturing plants in other countries. This distinction is relevant because vendors understandably do most of their clinical projects close to home and have limited research departments in their foreign subsidiaries. The change of Connaught Laboratories to a multinational company could decrease the number of clinical trials it sponsors in Canada. This would be unfortunate because Connaught so far has been the major sponsor of vaccine trials in Canada.

The investigators who participate in field studies have included both public health physicians and academics from various disciplines. Until recently, vaccine trials have been uninviting for academics. Companies exerted extensive control over protocol design, trial management and data analysis, leaving little to challenge the intellect. Trials often emphasized multicentre designs for perceived 'political' credit rather than for scientific necessity. The ensuing small roles per centre and the limited credit available through multi-author publications further diminish interest. The token studies undertaken by foreign vaccine makers to support licensure applications were typically of limited scientific value, being late phase 2 protocols duplicating work already completed (and often published). Investigators who sought grant funding from scientific agencies to address post licensure questions quickly discovered that agencies had a 'let the companies fund it' attitude.

This is not to say that participation in vaccine studies cannot be worthwhile for academics. Certainly the need for additional evaluators is unprecedented at the global level given the pace of new vaccine development and the multiplicity of competing products. In the United States, a closer interaction between academic groups and manufacturers evolved in the past decade to mutual advantage; this was facilitated by the large number of United States based companies and their proximity to university centres. A giant assist came from a National Institutes of Health (NIH) program to sponsor six major 'vaccine centres' in various American universities. Each has a specific focus (such as enteric, respiratory or pediatric vaccines) and was given the wherewithal to pursue both basic vaccine development and clinical evaluation, including sophisticated measurements of immunity. Both the NIH-funded and other specialized academic groups had considerable expertise to offer to manufacturers in return for major responsibility for clinical trials design and completion. Shifting the locus of control had mutual advantages: investigators could function productively as clinical scientists and gain appropriate academic recognition while manufacturers obtained high quality studies from respected outside authorities. The NIH vaccine centres were ideally suited to undertake independent comparisons of products, including standardized laboratory testing, serving the needs of consumers and health authorities in an innovative manner.

In short, the relationship between manufacturer and investigator is negotiable if the latter can offer special expertise. In a general climate of shrinking research agency support and growing respect for university-industry collaboration, the opportunity to work productively with manufacturers has been welcomed by many American academics.

\section{THE VANCOUVER EXPERIMENT}

In 1988 we looked critically at vaccine evaluation research in Canada and became concerned about its future. Involvement of academic investigators and foreign manufacturers was limited. Despite the abundance of new products, the unprecedented need for evaluators, the many intellectual challenges and the legislative requirement for companies to invest in more research in Canada, no movement was evident. There was an acute need for leadership in seeking more appropriate roles for academics and in facilitating company-funded and other vaccine research in Canada. Without some decisive changes, we feared that opportunities would be lost and vaccines would come to market with little or no Canadian testing beforehand.

We undertook an experiment towards finding an appropriate solution based on the concept of NIH vaccine centres. Our plan was to assemble a team of investigators and skilled managers with broad capabilities, particularly in the areas of clinical trials design, management and analysis. Lacking access to NIH, we would seek private establishment support. Since that could not amount to millions of dollars per year, 
we would forgo the infrastructure for basic research towards vaccine development (except as already existed) and concentrate instead on clinical evaluation. We would also forgo the limited focus of NIH centres, substituting instead a broad focus that would provide access to a wide range of funding sources. Since peer review is a key academic talisman, we would try to achieve a 40:60 balance between scientific agency and company funded projects. We chose from the outset to remain independent of industry, undertaking projects from all companies but accepting no infrastructure support or personal income from them. That impartiality would permit us to speak more effectively on behalf of vaccine consumers.

The key innovation was a team approach to facilitating vaccine research. We would gather the dozen or more local professionals with particular interest in vaccines into a collaborative group with broad collective expertise. We would encourage similar talent-pooling nationally to create a brains trust attractive to manufacturers and convincing to reviewers. At the operations level we would develop an organization to support clinical trials. The availability of skilled managers, coordinators and field staff would make it possible for individual investigators to undertake multiple projects simultaneously. Indeed, to keep such a team busy would require 10 or more projects annually. That level of activity offers economies of scale that would permit us to be highly cost competitive. A mixed venue of projects would diversify funding sources and offer more stability from year to year than would be possible with attachment to one company or one type of vaccine.

Our goal was to develop a centre of excellence for vaccine trials that would be recognized internationally. Our expectation was for investigators to exert scientific control over most (if not all) projects, restoring their academic nature. Our hope was to gain access to company-sponsored projects that would otherwise have been done outside Canada, with early phase 2 studies a priority. We guessed that it would take five years of gradual attitudinal shifts and trust-building before we could tell if the experiment would work.

As of the three-year mark (December 1991), there has been substantial progress. Over 30 studies have been undertaken or completed, with budgets totalling over $\$ 2,000,000$. Thirteen studies have been grantfunded (17 industry-funded); seven were multicentre trials coordinated by us. The team of local investigators included five different principals (one in Vancouver on sabbatical) and six co-investigators, most being medical health officers. Their cooperation in providing access to children attending public immunization clinics has permitted efficient recruiting for large trials. The support team at the centre includes 20 employees encompassing an administrative manager, assistant and secretary, a data manager and data entry staff, several coordinators and research assistants, a pool of experienced research nurses and a serology technician. We have also been privileged to work with two independent field research teams with long experience with vaccine trials.

Nationally, we have been able to collaborate with nine other investigators distributed nationwide. These included peers in infectious diseases, as well as colleagues in government, particularly at the Laboratory Centre for Disease Control. The range of interactions has been as diverse as training research nurses for new investigators, helping others to prepare appropriate budgets, and receiving help with project designs and specialized serological tests.

The best news is in the category of 'academic fulfillment'. Among the 17 industry funded studies, eight were planned totally and five partially by our group. Four different manufacturers are included in these figures. The attraction of an efficient, reliable organization proved to be powerful. Companies needed little convincing of the value to them of high quality studies performed by outside investigators. The Bureau of $\mathrm{Bi}$ ologics was strongly supportive of such arrangements, without which our initiatives would have been abortive. Major granting agencies were persuaded of the need to fund certain types of vaccine studies, opening the door wider for all investigators. Publications totalled 19 but some papers are still in press and some studies have not concluded (see Bibliography).

The mixture of project themes was eclectic, as intended. A partial list includes large scale studies of rubella immunization, 12 studies involving three different $H$ influenzae $\mathrm{b}$ conjugate vaccines, three studies of influenza vaccination, four studies of reactions to diphtheria-pertussis-tetanus vaccines and their basis, a study of parent education methods and a pilot study of a country-wide, hospital-based network for surveillance of vaccine adverse reactions.

Despite this level of activity, the centre has not been able to achieve long term financial self-sufficiency. Projects employ many of the staff continuously but there remain planning, staff development and administrative costs that cannot be recovered from projects. To date such overhead expenses have been met by an establishment grant from our host hospital but this is finite, keeping us searching for an NIH-like sponsor.

A potential concern in developing a vaccine centre is to draw opportunities away from vaccine-oriented colleagues in other provinces. We have tried hard to avoid this. Many industry sponsored projects listed above would not have been done in Canada had we not been able to compete effectively for them. This included the prospect of three large, multicentred phase 3 trials. While they were not funded, the planning process required recruitment of colleagues into a Canada-wide network. As the exercise was repeated we all realized that the pediatric infectious diseases community has a remarkable willingness to work together on major pro- 
jects. This is a rare asset that we and others continue to market on behalf of the group. While megaprojects are too few to sustain a large network, there have been positive developments nonetheless. Some colleagues have been 'turned on' to vaccine studies and will continue to participate. Others are following our lead in developing vaccine centres, with our strong encouragement and full cooperation. As we reach our capacity, prospects for some industry-sponsored projects are being redirected to interested colleagues in other centres to keep the work in Canada.

\section{CONCLUSIONS}

Unprecedented activity is going on in vaccine development, creating great opportunities for clinical evaluations. While not always the case, approaches exist to make involvement in prelicensure trials academically rewarding. Opportunities for such involvement tend to be limited by the small size of the Canadian marketplace and the limited number of Canadian-based vaccine companies. This tendency can be offset if Canadian investigators are prepared to stand tall and stand together, offering collectively the powerful inducements of special skills and efficiency. Just as Finland has played a disproportionately large role in vaccine studies in the 1980 s, Canadian investigators can do likewise in the 1990s. The opportunity is ours to seize, collectively.

ACKNOWLEDGEMENTS: The authors are both Career Investigators of British Columbia's Children's Hospital Foundation (which also provided the establishment grant for the Centre). The authors are deeply grateful for the support received from Dr John Furesz as Director of the Bureau of Biologics and from Gordean Bjornson, Administrative Director of the Centre.

\section{BIBLIOGRAPHY}

1. Meekison W, Arcand T, Scheifele D, Humphreys G. Local adverse reactions to adsorbed DPT vaccine - Surrey, British Columbia. Can Dis Week Rep 1989;15:73-4.

2. Scheifele DW, contributing author. Canadian Immunization Guide, 3rd edn. Health and Welfare Canada, 1989.

3. Meekison W, Scheifele D, Grace M, et al. Postmarketing surveillance of adverse events following haemophilus b diphtheria toxoid conjugate vaccine - Surrey, British
Columbia. Can Dis Week Rep 1989;15:143-5.

4. Scheifele DW, Bjornson GL, Arcand T, Jacobsen K. Antigenuria following receipt of haemophilus $\mathrm{b}$ diphtheria toxoid conjugate vaccine. Pediatr Infect Dis $\mathrm{J}$ 1989;8:887-8.

5. Scheifele DW, Bjornson GL, Johnston J. An evaluation of the adverse effects of influenza vaccine in hospital personnel. Can Med Asoc J 1990;142:127-30.

6. Scheifele DW, Grace M, Meekison W, et al. Haemophilus b diphtheria toxoid conjugate vaccine - postmarketing study. Can J Pediatr 1990;2:4-6.

7. Meekison W, Hutcheon M, Guasparini R, et al. Postmarketing surveillance of adverse reactions to ProHIbiT vaccine in British Columbia. Can Med Assoc $J$ 1989;141:927-9.

8. Scheifele DW. An update on haemophilus b vaccines. Med North Am 1990;11:1308-11.

9. Scheifele DW. Immunization advice for special situations. Med North Am 1990;11:1341-8.

10. Tingle AJ, Pot KH, Yong FP, Puterman ML, Hancock EJ. Kinetics of isotype-specific humoral immunity in rubella vaccine-associated arthropathy. Clin Immunol \& Immunopathol 1989:53:S99-106.

11. Scheifele DW, Meekison W, Grace M, et al. Adverse reactions to the preschool (fifth) dose of adsorbed diphtheria-pertussis-tetanus vaccine in Canadian children. Can Med Assoc J 1991;145:641-7.

12. Al-Mazrou A, Scheifele D, Soong T, Bjornson G. A blinded, controlled comparison of adverse effects following whole or split virion influenza vaccines administered to hospital personnel. Can Med Assoc J 1991;145:213-8.

13. Scheifele D, Bjornson G, Barreto L, Meekison W, Guasparini R. Controlled trial of Haemophilus influenzae type $\mathrm{b}$ diphtheria toxoid conjugate combined with diphtheria tetanus and pertussis vaccines, in 18 month old children, including comparison of arm versus thigh injections. Vaccine 1992;10:455-60.

14. Herrman D, Hamilton R, Barington T, et al. Quantitation of human IgG subclass antibodies to Haemophilus influenzae type b capsuler polysaccharide: Results of an international collaborative study using enzyme immunoassay methodology. J Immunol Meth 1992;148:101-14.

15. Mitchell LA, Zhange T, Ho M, et al. Characterization of rubella virus-specific antibody responses using a new synthetic peptide-based enzyme-linked immunosorbent assay. J Clin Microbiol 1992;30:1841-7.

16. Zhan T, Mauracher CA, Mitchell LA, et al. Detection of rubella-specific IgG, IgM and IgA antibodies by immunoblot assays. J Clin Microbiol 1992;30:824-30.

17. Scheifele D, Bjornson G. Evaluation of inactivated hepatitis A vaccine in Canadian adults over 40 years of age. Can Med Assoc J. (In press)

18. Mitchell LA, Zhang T, Tingle AJ. Differential antibody responses to rubella virus infection in males and females. $\mathrm{J}$ Infect Dis 1992;166:1250-65. 


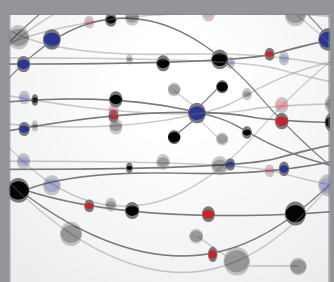

The Scientific World Journal
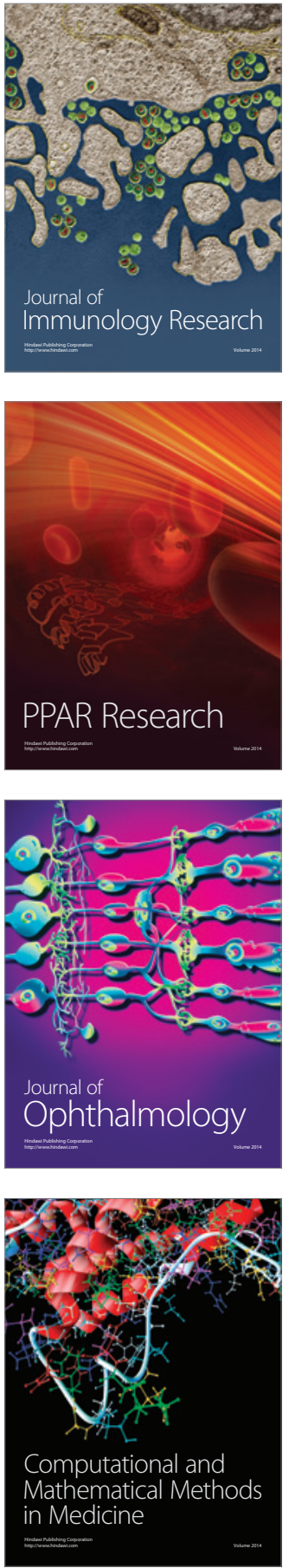

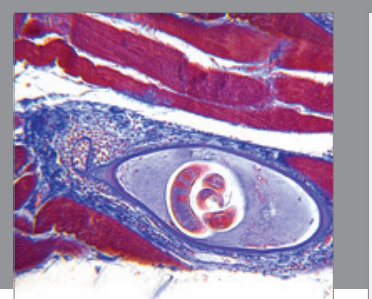

Gastroenterology Research and Practice

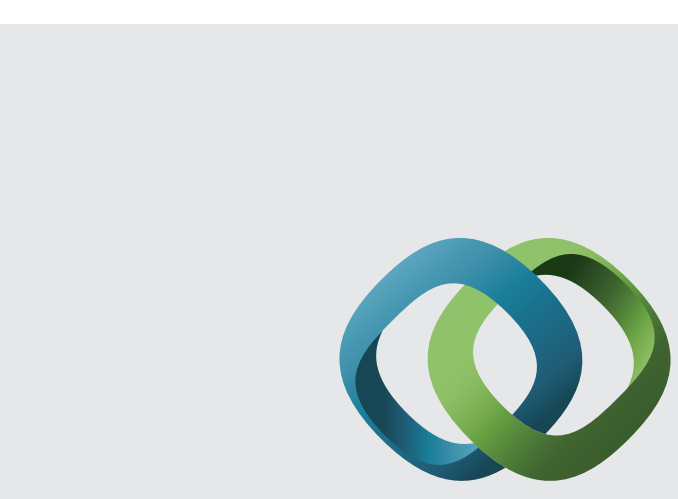

\section{Hindawi}

Submit your manuscripts at

http://www.hindawi.com
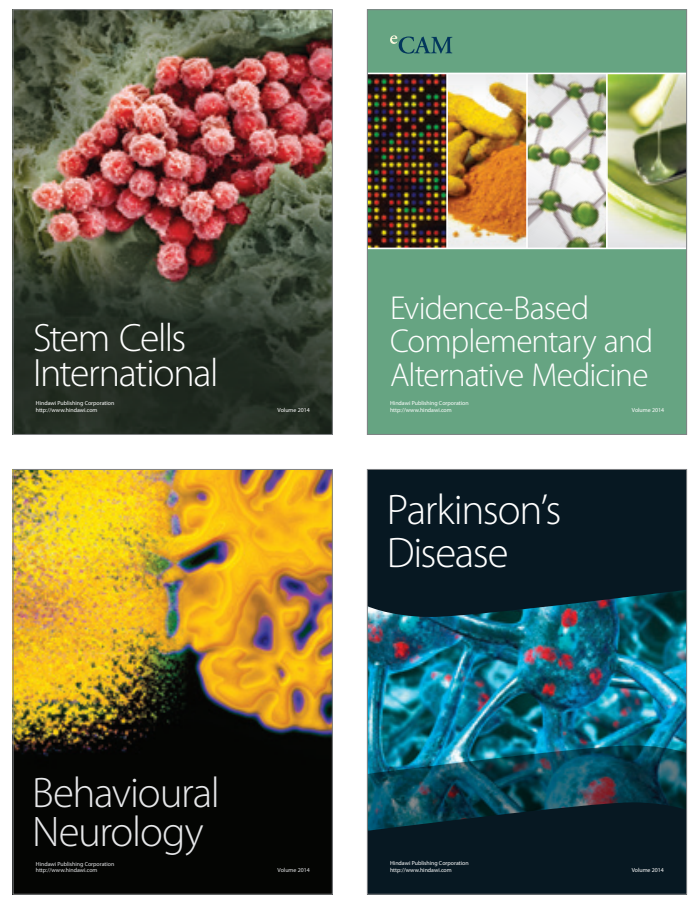
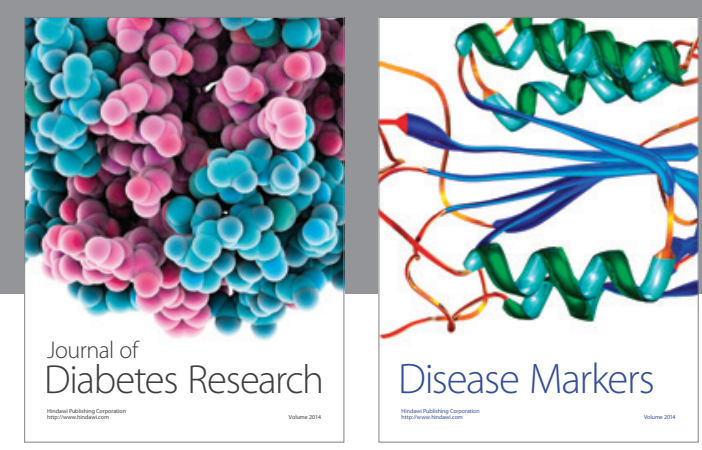

Disease Markers
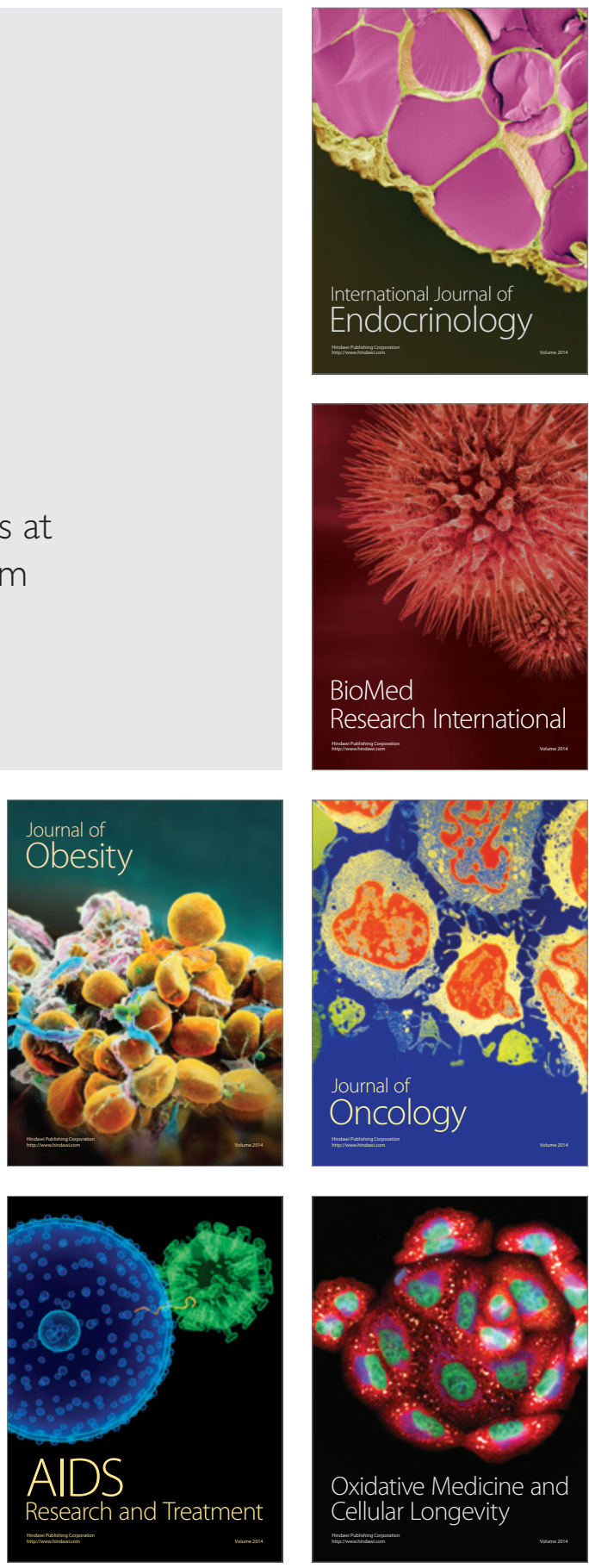